\title{
Isolation and characterization of Acetobacter aceti from rotten papaya
}

\author{
J. Kowser ${ }^{*}$, M. G. Aziz and M. B. Uddin \\ Department of Food Technology and Rural Industries, Bangladesh Agricultural University, Mymensingh-2202, \\ Bangladesh, *Email: jerinkowser89@gmail.com
}

\begin{abstract}
The present study was concerned with the isolation and characterization of Acetobacter aceti from rotten papaya for vinegar production. The samples were inoculated in sterilized GYC standard media and then incubated at $30^{\circ} \mathrm{C}$ for 48 hours. Successive subculture was performed to screen out the strains. In Gram's staining, the morphology of the isolated bacteria exhibited pink, small rod shaped single, pair and chain in arrangement, in the hanging drops technique, all the isolates revealed motile. Biochemical tests were performed by fermentation of five basic sugars by producing both acid and gas bubbles in Durham tube. All of the isolates were Indole, Voges-Proskauer (VP) and Oxidase negative, Methyl Red (MR) and Catalase positive. The growth rate of isolated strain was optimized by weighing dry cell and turbidity at $600 \mathrm{~nm}$ at different concentrations of dextrose (1\%, 5\% and 10\%). Ten (10) percent dextrose solution showed rapid growth and higher cell mass than $5 \%$ and $1 \%$ solution respectively. Acidity of the media gradually increased from $0.102 \%$ to $2.18 \%$ from day 0 to day 7 and $\mathrm{pH}$ of the media decreased from 6.8 to 5.5 during the period. This protocol was successful for enriching Acetobacter aceti, which was essential for vinegar production.
\end{abstract}

Keywords: Rotten Papaya, Acetobacter aceti, GYC culture media, Morphological and Biochemical assessment

\section{Introduction}

Acetic acid bacteria (AAB) is a group of gram-negative, aerobic and motile rods that carry out incomplete oxidation of alcohol and sugars, leading to the accumulation of organic acids as end products. These bacteria are heterogeneous assemblage organisms (Sokollek et al., 1998; Madigan et al., 2008). Acetic acid bacteria are commonly associated with some fruits such as grape, apple, papaya etc and are normally present in most deteriorated fruits (Gullo and Giudici, 2008). Among the most important acetic acid bacteria, the strains of genus Acetobacter are mainly involved in vinegar production. Acetobacter is a gram negative, obligate aerobe coccus or rod shaped bacterium with the size of 0.6--0.8 X $1.0-4.0 \mu \mathrm{m}$, nonmotile or motile with peritrichous flagella, catalase positive and oxidase negative biochemically. Acetobacter use ethanol as carbon source preferably and are increased during the wine fermentation processing (Joyeux et al., 1984; Drydale and Fleet, 1985; Kadere et al., 2008; Toit and Lambrechts, 2002). They are characterized by the ability to convert ethanol to acetic acid in the presence of oxygen. Acetobacter species can be easily distinguished in the laboratory by the growth of colonies on a medium containing about $7 \%$ ethanol and enough calcium carbonate to render it partially opaque. When Acetobacter colonies form enough acetic acid from the ethanol, the calcium carbonate around the colonies dissolves, forming a very distinct clear zone.

Acetobacter aceti has not yet been reported as a pathogenic microbe to humans or animals. It does not produce any toxins, enzymes, or any viruses that harm any human or animal. It is ubiquitous in nature and there is contact of this bacterium on all animals on a frequent basis. Acetobacter aceti is known to cause rotting and browning discoloration in fruits such as apples, pears and citrus products. The significance of Acetobacter aceti in food technology in Bangladesh having low agricultural production and rapidly increasing population makes the production of food grade vinegar extremely important.

Papaya is a good source of Acetobacter aceti in Bangladesh since it is a very common fruit and its production is increasing day by day due to the climate of Bangladesh. It is a highly perishable fruit with very poor keeping quality since it contains approximately $90 \%$ moisture and its skin is thin. Post-harvest loss of this fruit is high; $40-100 \%$ losses have been reported in developing countries (Teixiera da Silva et al., 2007). This waste can be reduced by making papaya vinegar. 
Considering all above mentioned facts, this study was undertaken (i) to identify and isolate potential acetic acid bacteria Acetobacter aceti from isolated strains, (ii) to assess the effect of carbon source on the growth of Acetobacter aceti and (iii) to optimize growth rate of Acetobacter aceti in producing acetic acid.

\section{Materials and Methods}

\section{Enrichment of samples}

GYC broth was used as enrichment medium. This is the medium used for the growth and maintenance of Acetobacter spp. having the formulation of glucose $100 \mathrm{~g} / \mathrm{L}$, calcium carbonate $20 \mathrm{~g} / \mathrm{L}$, yeast extract 10 $\mathrm{g} / \mathrm{L}$ and distilled water $1000 \mathrm{ml}$. $\mathrm{pH}$ was adjusted with $0.1 \mathrm{~N} \mathrm{NaOH}$ to 6.8 for masking the growth of other microorganisms (Zahoor et al., 2006).

\section{Inoculation and incubation of sample}

Spread plate technique was followed throughout this study. Diluted samples of $0.1 \mathrm{ml}$ were transferred with sterile pipette and spreaded on GYC agar plates with the help of sterile bent rod in a sterile condition inside the laminar flow. All the GYC plates were incubated in an incubator at $30^{\circ} \mathrm{C}$ for 48 hours. Then single morphology well-formed colonies were isolated and sub cultured by streak plate technique on GYC agar plates to check its purity and incubated at $30^{\circ} \mathrm{C}$ for 48 hours.

\section{Gram's staining}

Gram's staining was performed for morphological characteristics and staining reaction (+ve, -ve) of isolated strains. The representative Acetobacter aceti colonies were characterized morphologically using Gram's stain.

\section{Biochemical examination of the isolates}

Sugar fermentation test: The carbohydrate fermentation test was performed by inoculating a loop full of nutrient broth culture of the organisms into the tubes containing five basic sugars e.g., dextrose, sucrose, lactose, maltose and mannitol, incubated for 24 hours at $37^{\circ} \mathrm{C}$. Acid production was indicated by the colour change reddish to yellow in the medium and the gas production was noted by the appearance of gas bubbles in the inverted Durham's tubes.

Methyl red (MR) test: The test was performed by inoculating a colony of the test organism in $0.5 \mathrm{ml}$ sterile glucose phosphate broth (as used in the VP test). After overnight incubation at $37^{\circ} \mathrm{C}$, a drop of methyl red solution was added.

Methyl-Red Voges-Proskauer (MR-VP) broth: A quantity of $3.4 \mathrm{~g}$ of Bacto MR-VP medium was dissolved in $250 \mathrm{ml}$ distilled water dispensed in $2 \mathrm{ml}$ amount in each test tube and then the tubes were autoclaved at $121^{\circ} \mathrm{C}$ for 15 minutes maintaining a pressure of $1 \mathrm{~kg} / \mathrm{cm}^{2}$. After autoclaving, the tubes containing medium were incubated at $37^{\circ} \mathrm{C}$ for overnight.

Indole test: For this test, $2 \mathrm{ml}$ peptone water was inoculated with a pure colony of bacterial culture under observation and incubated at $37^{\circ} \mathrm{C}$ for 48 hours after which $0.5 \mathrm{ml}$ Kovac's reagent was added, shaked well and examined after 1 minute. A red colour in the reagent layer indicated positive test (Cheesbrough, 1985).

Motility test: This test was performed in Hanging drop slide. One drop of distilled water was taken in the glass slide and a single colony was taken from the GYC agar with a small tip and mixed it very well. Then it was observed under a microscope using immersion oil.

Catalase test: A small colony of good growth pure culture of test organism was smeared on a slide. One drop catalase reagent $\left(3 \% \mathrm{H}_{2} \mathrm{O}_{2}\right)$ was added on the smear. The slide was observed for bubble formation (Cheesbrough, 1985). 
Oxidase test: One or two drops oxidase reagent were placed over the bacteria on a swab to moisten the filter paper. The results were recorded by the development of blue colour on the bacterial colony swab within 30 seconds by following the method of Ashecraft et al. (2001).

Cell growth by dry cell wt. methods: At first a filter paper was taken and placed in an oven (at $65 \pm$ $\left.5^{\circ} \mathrm{C}\right)$ for $1 \mathrm{~h}$. Then the weight $\left(\mathrm{W}_{1}\right)$ of the filter paper was taken. After incubation, the biomass was filtered through the filter paper. The filter paper with biomass was placed in an oven (at $65 \pm 5^{\circ} \mathrm{C}$ ) for $24 \mathrm{~h}$ and weighed $\left(\mathrm{W}_{2}\right)$ after drying. The gain in weight was taken as the cell growth of the Acetobacter aceti. The readings were taken after incubating the test tube for every $24 \mathrm{~h}$ over 9 days and the cell growths were calculated as follows:

Dry cell wt. $\left(\right.$ cell growth, $\left.\frac{g}{L}\right)=\frac{\left(w_{2}-w_{1}\right)}{5} \times 1000$

Cell growth by absorbance: The absorbance of liquid culture media of the Acetobacter aceti for every 24 hours over 9 days was observed using spectrophotometer at $600 \mathrm{~nm}$.

pH and Acidity of media: The pH of the sample was measured by using PERKINFLMER Metrion$\mathrm{V} \mathrm{pH}$ meter at the ambient temperature. Titratable acidity was determined using AOAC (2005) method.

Maintenance of stock culture: Pure culture of isolated Acetobacter aceti organisms were kept in stock culture. $30 \%$ sterile buffered glycerin was made by mixing 50 parts pure glycerin and 50 parts PBS. Then a loopful of thick bacterial spore was mixed with $50 \%$ sterile buffered glycerin in small vials (cryo vials) and was preserved at $-50^{\circ} \mathrm{C}(\mathrm{WHO}, 1973)$.

\section{Results and Discussion}

\section{Cultural characteristics of isolated strains}

Four colonies were selected from the culture plate on the basis of their differences of cultural characteristics designated as $\mathrm{S}_{1}, \mathrm{~S}_{2}, \mathrm{~S}_{3}$ and $\mathrm{S}_{4}$. The cultural (colony) characteristics of isolated strains are described and represented in Table $1 . S_{1}, S_{2}$ and $S_{4}$ were circular in shape while the other was irregular in shape. The shape of the isolated sample varied from 1 to $3 \mathrm{~mm}$. Isolated samples showed different types of colony color such as white, off white, creamy white and pinkish white. Opacity and consistency of all the colonies were opaque, pale and viscid respectively. $S_{1}$ and $S_{4}$ were found convex elevation while $\mathrm{S}_{2}$ and $\mathrm{S}_{3}$ were raised. The surfaces of the isolates were smooth, moist and oily. Similarly Zahoor et al. (2006) founds that colonies were smooth, small, medium, big and white, off white, pale, spherical, raised, convex, spheroid, star shaped, rough, crinkled and flat.

Table 1. Cultural characteristics of isolated strains

\begin{tabular}{cccccccccc}
\hline Isolate & Shape & Size $(\mathbf{m m})$ & Colony color & Opacity & Elevation & Surface & Consistency \\
\hline $\mathrm{S}_{1}$ & Circular & $<3$ & White & Opaque & Convex & Smooth moist & Viscid \\
$\mathrm{S}_{2}$ & Circular & $1-2$ & Off white & Pale & Raised & Smooth & Viscid \\
$\mathrm{S}_{3}$ & Irregular circular & $2-3$ & Creamy white & Pale & Raised & Smooth oily & Viscid \\
$\mathrm{S}_{4}$ & Circular & $1-3$ & Pinkish white & Opaque & Convex & Smooth & Viscid \\
\hline
\end{tabular}

\section{Gram's staining}

Morphological and staining characteristics of bacteria recorded from papaya by Gram's staining are presented in Table 2 and Fig. 1 . All the isolates $\left(S_{1}, S_{2}, S_{3}\right.$ and $\left.S_{4}\right)$ were rods, ellipsoidal, roundish and bacilli squat in shape, single, paired or chain in cell arrangements and Gram's staining reactions were gram negative and pink color. 
Table 2. Morphological and staining properties of isolated strains

\begin{tabular}{|c|c|c|c|c|c|}
\hline \multicolumn{5}{|c|}{ Characteristics } & \multirow[b]{2}{*}{ Motility } \\
\hline Isolates & Shape & Arrangements & $\begin{array}{l}\text { Gram's staining } \\
\text { reaction }\end{array}$ & colour & \\
\hline $\mathrm{S}_{1}$ & Small rods shape & Single, paired or in short chain & $(-)$ ve & pink & + \\
\hline $\mathrm{S}_{2}$ & Ellipsoidal, rods & Single or chain & $(-)$ ve & pink & + \\
\hline $\mathrm{S}_{3}$ & Rods, roundish & Single, in paired or chain & $(-)$ ve & pink & + \\
\hline $\mathrm{S}_{4}$ & Rods, squat bacilli & Single, paired or in chain & $(-)$ ve & pink & + \\
\hline
\end{tabular}

$(+)=$ positive,$(-)=$ negative

These findings agreed with several authors (Zahoor et al., 2006; Loganathan, 1999). Similar results were also obtained by Yamada et al. (1999), Lisdiyanti et al. (2001), Ashecraft et al. (2001) who also isolated Acetobacter spp. and found that morphologically these are gram negative rods and Kadere et al. (2008) found Acetobacter aceti was motility positive.

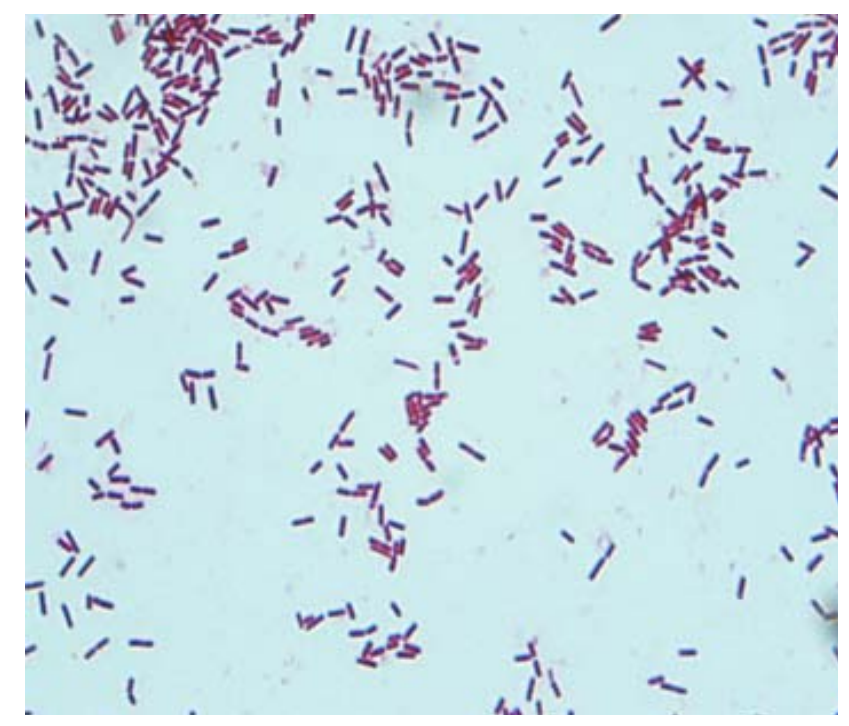

Fig 1. Gram's staining of isolate

\section{Results of biochemical tests}

Results of sugar fermentation: All the isolates fermented five basic sugars (dextrose, sucrose, lactose, maltose and mannitol) with the production of acid and gas. They fermented glucose and sucrose with the production of acid and gas within 48 hours of incubation but negative reaction was observed for lactose, maltose and mannitol (Table 3, Fig. 2). This result was similar to that of Aydin and Aksoy (2009). Kadere et al. (2008) also reported that glucose, sucrose, mannose were positive and lactose, maltose, mannitol were negative.

Results of other biochemical tests: All the isolates were shown positive for methyl-red and catalase test and negative for VP, Indole and oxidase test (Table 3, Fig. 3, 4, 5). This was preciously suggested by a number of scientists (Sim et al., 2001; Aydin and Aksoy, 2009). According to these authors, positive results were shown for methyl red and Indole production and negative for Voges-Proskauer. Ashecraft et al. (2001) who observed that Acetobacter aceti had the ability of catalase production and showed negative results for oxidase production. 
Table 3. Biochemical characteristics of Acetobacter aceti

\begin{tabular}{lc}
\hline \multicolumn{1}{c}{ Biochemical tests } & Results \\
\hline 1. Fermentation reaction with five basic sugars \\
a. Dextrose \\
b. Maltose \\
c. Lactose \\
d. Sucrose & AG \\
e. mannitol & - \\
& AG \\
2. Indole production test & - \\
3. MR test & + \\
4. VP test & - \\
5. Catalase test & + \\
6. Oxidase test & - \\
\hline
\end{tabular}

$(+)=$ positive; $(-)=$ negative; $M R=$ Methyl Red VP = Voges Proskauer; AG= Acid and gas

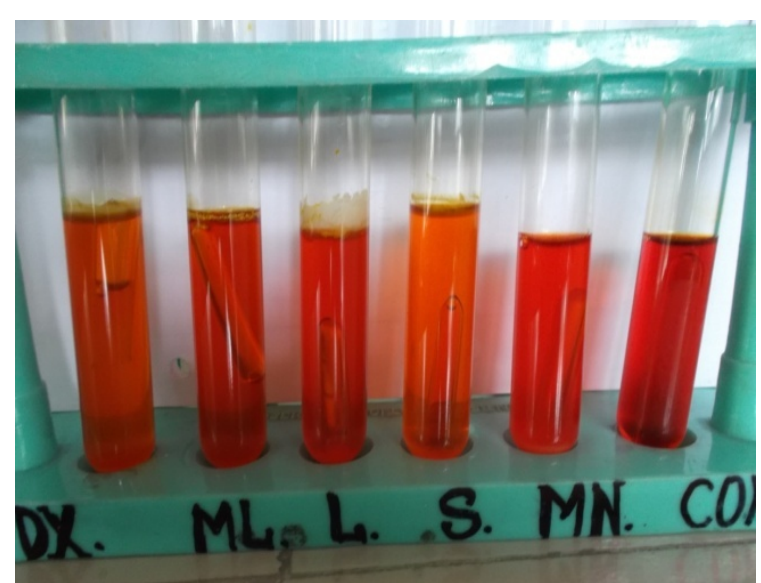

Fig. 2. Results of sugar fermentation tests

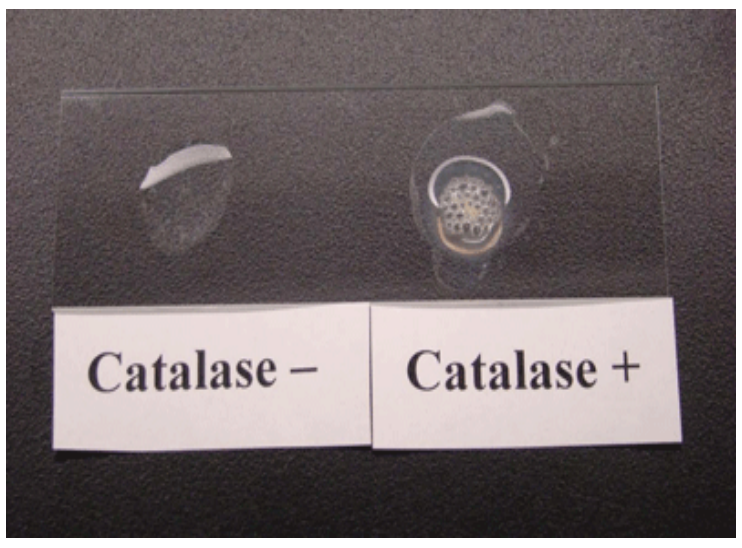

Fig. 4. Catalase test of isolate $S_{1}$

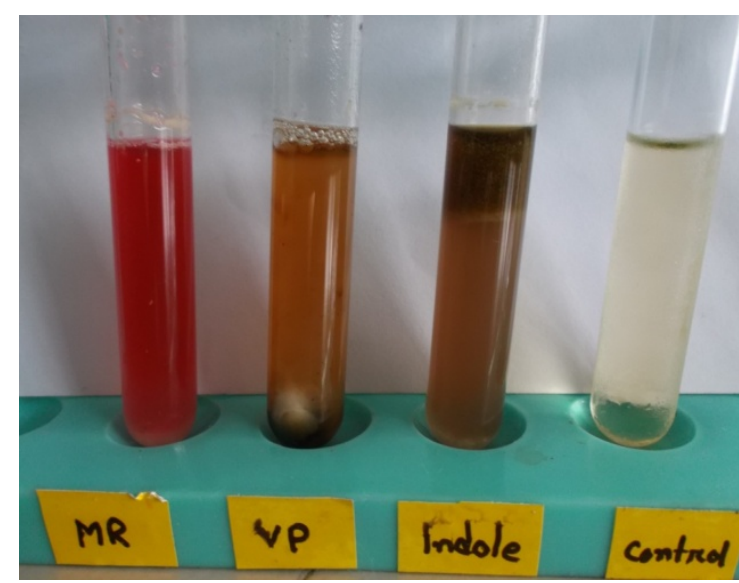

Fig. 3. Results of Methyl Red, Voges-Proskauer and Indole tests

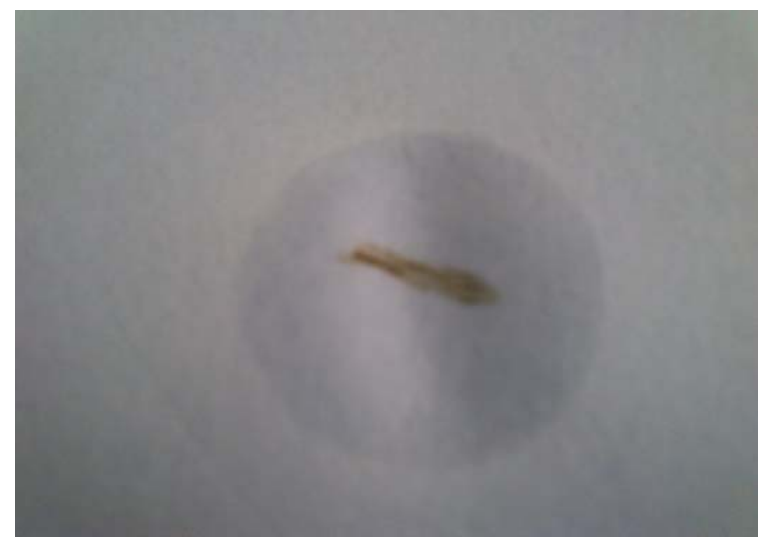

Fig. 5. Oxidase test of isolate $\mathrm{S}_{1}$ 
Growth rate of Acetobacter aceti by dry cell weight: The growth rate of Acetobacter aceti was optimized by monitoring the growth kinetics in terms of dry cell weight at different concentration of dextrose $(1 \%, 5 \%$ and $10 \%)$ and the absorbance of liquid broth media. From different concentration of dextrose it was observed that at $10 \%$ dextrose solution, Acetobacter aceti showed rapid growth and higher cell mass than that of at $5 \%$ and $1 \%$ respectively as shown in Figure 6 . The highest cell growth rate was found on day 3 irrespective of dextrose concentrations. After then cell growth rate drastically reduced and it remained almost unchanged up to 7 days. It means that the growth of Acetobacter aceti in three media containing $1 \%, 5 \%$ and $10 \%$ dextrose respectively were followed log phase within three days of incubation period and from 3 to 7 the phase seems to be stationery.

According to Chowdhury (2010) the growth rate of Rhizopus oryzae was optimized by dry cell weight at different concentration of dextrose $(1 \%, 5 \% \& 10 \%)$. It was observed that $10 \%$ dextrose solution showed rapid growth on second day of incubation.

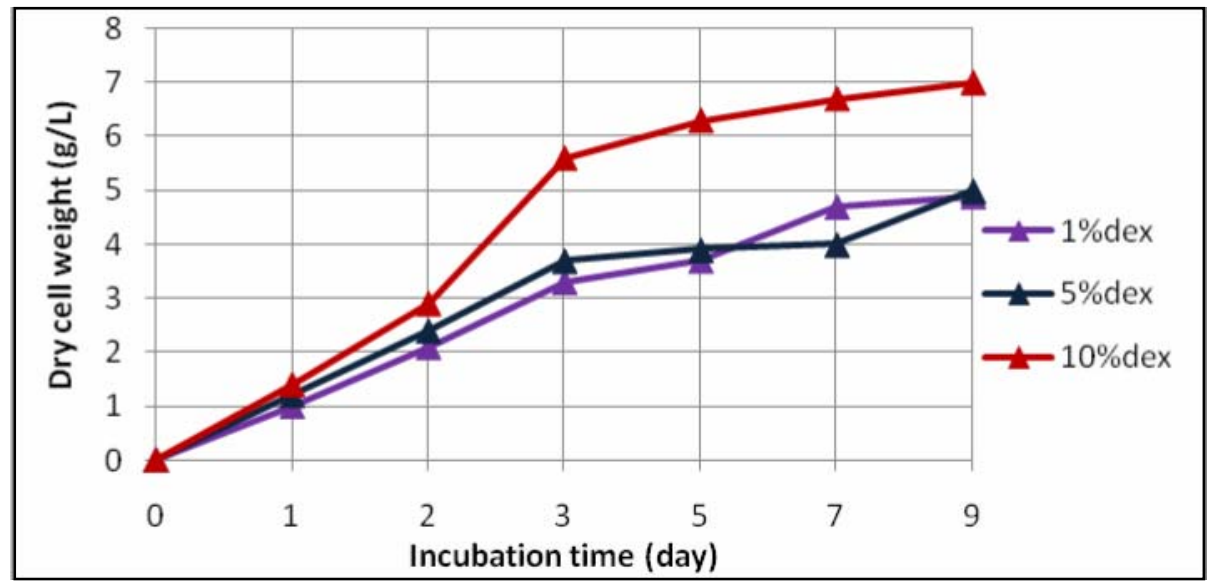

Fig. 6. Growth curve of isolate $\mathrm{S}_{1}$ by dry cell weight

Growth rate of cells of Acetobacter aceti by turbidity of broth: The inoculation of pure Acetobacter strain in culture media showed the growth of cells in liquid media. Absorbance at $600 \mathrm{~nm}$ of the culture medium is used to optimize the growth rate of Acetobacter aceti in liquid broth as shown in Figure 7 . It was observed that the growth rate of change was highest on second day of incubation. After then cell growth rate was decreased upto the culturing period (9 days).

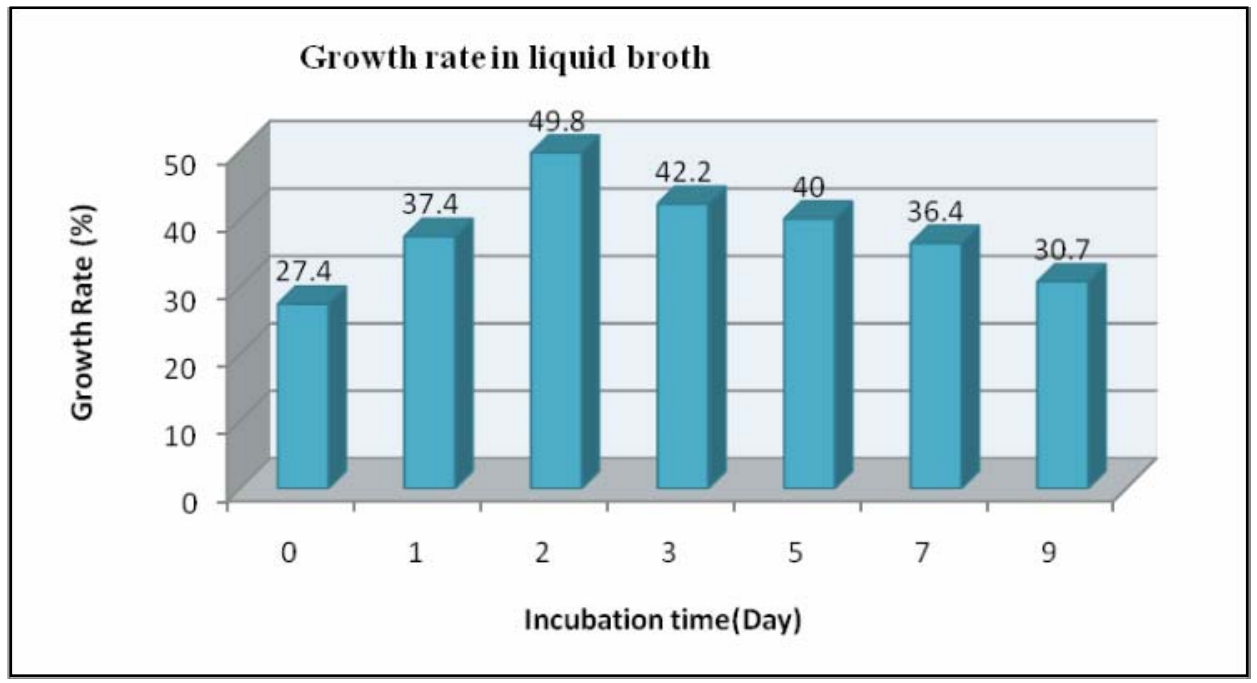

Fig. 7. Growth rate of isolate $S_{1}$ in liquid broth 
Production of acidity during cell growth: Acidity and $\mathrm{pH}$ of the culture media was also recorded up to seven days as shown in Figure 8. It was observed that acidity of the media was gradually increased from $0.102 \%$ to $2.18 \%$ during 1-7 days incubation period at $30^{\circ} \mathrm{C}$ and $\mathrm{pH}$ of the media was decreased correspondingly from 6.8 to 5.5. According to Maal et al. (2010) it was observed that the inoculation of Acetobacter strain to industrial culture media showed high acetic acid productivity after 1 to 6 days incubation at $30^{\circ} \mathrm{C}$ and $100 \mathrm{RPM}$ aeration speed. The acidity percent after 1 to 6 days incubation were $2.35 \%$ to $8.53 \%$ respectively.

Strain showed $2.18 \%$ acetic acid production after one week incubation without agitation and aeration that is a very good characteristic in producing vinegar in a short period of time in comparison to vinegar manufacturing time of acetic acid bacteria that is 14-30 days routinely (Toit and Lambrechts, 2002).

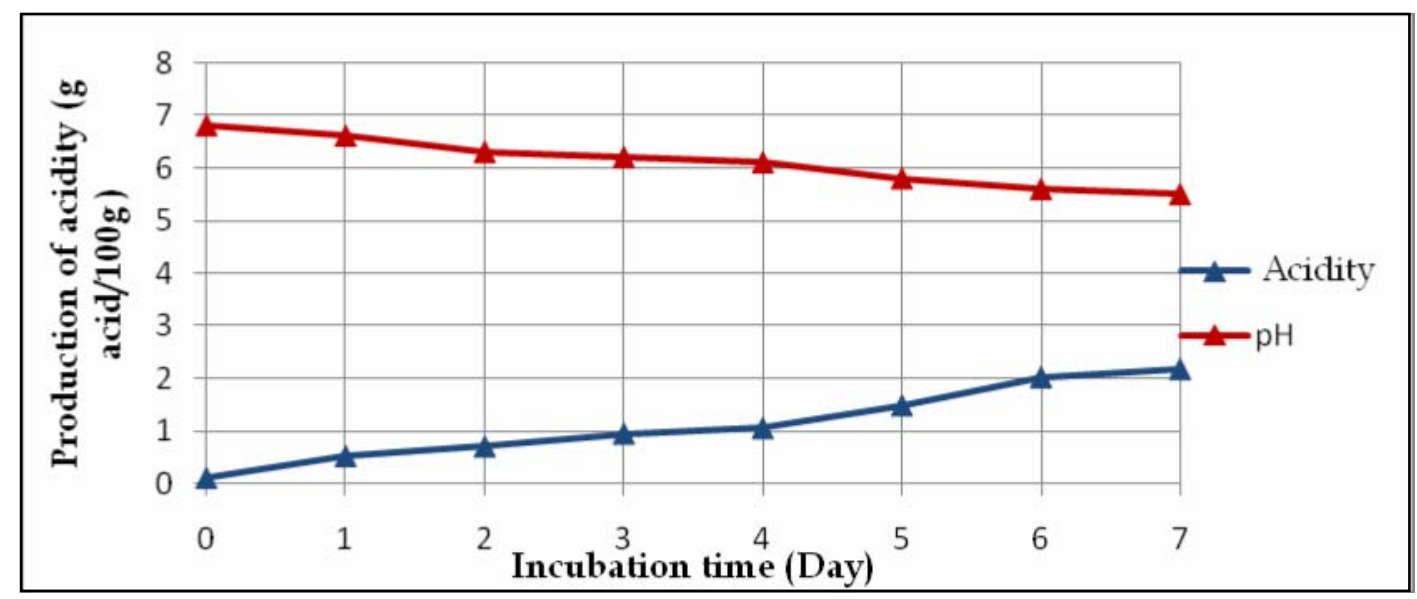

Fig. 8. Production of acidity and reduction of $\mathrm{pH}$

It may be concluded that it is necessary to develop pure vinegar cultures for vinegar production so that the use of synthetic vinegar may be avoided as it is prohibited in most countries. Keeping in view all of these points, the present work was conducted to isolate a pure culture of Acetobacter aceti and the maintenance of this culture. This strain could be a potential strain for production of vinegar type with a new and desirable taste. Papaya vinegar can be used to utilize the spoilage of this fruit as substrate to preserve the bioenvironment from food spoilage contaminations.

\section{References}

AOAC. 2005. Official methods of analysis. Association of Official Analytical Chemists. Fifth edition, Washington DC.

Ashecraft, E., Condron, S., Farsaii, P. 2001. "Acetobacter", available at: www.wam.umd.edu/asmith/emsarapay/intro.html.

Aydin, A.Y., Aksoy, D.N. 2009. Isolation of cellulose producing bacteria from wastes of vinegar fermentation. World Congress on Engineering and Computer Science (I), 978-988.

Cheesbrough, M. 1985. Medical laboratory manual for tropical countries. Microbiology. English language Book Society, London. pp. 400-480.

Drydale, G.S., Fleet, G.H. 1985. Acetic acid bacteria in some Australian wines. Food Technol., 37, 17-20.

Giudici, P., Rinaldi, G. 2007. A theoretical model to predict the age of traditional balsamic vinegar. J. Food Eng., 82, $121-127$.

Gullo, M., Giudici, P. 2008. Acetic acid in traditional balsamic vinegar, phenotypic traits relevant for starter cultures selection. Int. J. Food Microbiol., 125, 46-53.

Joyeux, A.S., Lafourcade, L., Gayon, R.P. 1984. Evolution of acetic acid bacteria during fermentation and storage of wine. Appl. Environ. Microbiol., 48(1), 153-156.

Kadere, T.T., Miyamoto, T., Oniang, K.R., Kutima, M.P., Njoroge, M.S. 2008. Isolation and identification of the genera Acetobacter and Gluconobacter in coconut toddy (mnazi). African J. Biotechnol., 7 (16), 2963-2971. 
Lisdiyanti, P., Kawasaki, H., Seki, T., Yamada, Y., Uchimura, T., Komagata, K. 2001. Identification of Acetobacter strains isolated from Indonesian sources, and proposals of Acetobacter sp. J. gen. Appl. Microbiol., 47(3), 119-31.

Loganathan, P. 1999. Isolation and characterization of the genetically distinct groups of acetobacter diazotrophicus from a new host plant eleusine coracana L. J. Appl. Microbiol., 87(1), 167-172.

Madigan, M.T., Martinko. M.J., Dunlap, V.P., Clark, P.D. 2008. Brock Biology of Microorganisms. Benjamin Cummings, USA.

Maal, B.K., Shafiei, R. 2010. Isolation and characterization of an Acetobacter strain from Iranian white-red cherry as a potential strain for cherry vinegar production in microbial biotechnology. Asian J. Biotechnol., 2(1), 53-59.

Maal, B.K., Shafiei, R., Kabiri, N. 2010. Production of apricot vinegar using an isolated Acetobacter strain from Iranian apricot. World Acad. Sci., Eng. Technol., 4(11), 162-165.

Mendes, F.A., Lea, C. 2004. Growth and fermentation patterns of Saccharomyces cerevisiae under different ammonium concentrations and its implications in winemaking industry. J. Appl. Microbiol., 97, 540-545.

Sim, C.K., Lee, S.K., Kim, H.D., Ryu, H.I., Lee, S.J. 2001. Studies on the acid tolerance of Acetobacter sp. isolated from persimmon vinegar. Korean J. Food Sci. Technol., 33(5), 574-581.

Sokollek, S.J., Hertel, C., Hammes, P.W. 1998. Cultivation and preservation of vinegar bacteria. J. Biotechnol., 60, $195-206$.

Teixiera, D.S.J.A., Rashid, Z., Nhut, D.T., Sivakumar, D., Gera, A., Souza, Jr.M.T., Tennant, P.F. 2007. Papaya (Carica papaya L.) biology and biotechnology. Tree and Forestry Sci. Biotech. 1 47-73.

Toit, J.W.D., Lambrechts, G.M. 2002. The enumeration an identification of acetic acid bacteria from South African red wine fermentations. Int. J. Food Microbiol., 74, 57-64.

WHO. 1973. Technical Report Series No. 530. General requirements for the sterility of biological substances. Geneva, World Health Organization.

Yamada, Y., Hosono, R., Lisdyanti, P., Widyastuti, Y., Saono, S., Uchimura, T., Komagata, K. 1999. Identification of acetic acid bacteria isolated from indonesian sources, especially of isolates classified in the genus Gluconobacter. J. gen. Appl. Microbiol., 45 (1), 23-8.

Zahoor, T., Siddique, F., Farooq, U. 2006. Isolation and characterization of vinegar culture (Acetobacter aceti) from indigenous sources. British Food J., 108(6), 429-439. 